\title{
Diclofenac versus Tramadol in the Treatment of Renal Colic: A Prospec- tive, Randomized Trial
}

\author{
Shaden Salameh*, ${ }^{*}$, Nurit Hiller ${ }^{2}$, Meir Antopolsky ${ }^{1}$, Fedaa Ghanem ${ }^{3}$, Yigaal Abramovitz ${ }^{4}$ and \\ Ruth Stalnikowics ${ }^{1}$
}

\author{
${ }^{1}$ Emergency Department - Hadassah Mount Scopus, Jerusalem, Israel \\ ${ }^{2}$ Radiology Department - Hadassah Mount Scopus, Jerusalem, Israel \\ ${ }^{3}$ Hadassah University Hospital, Jerusalem, Israel \\ ${ }^{4}$ Internal Medicine Department - Hadassah Mount Scopus, Jerusalem, Israel
}

\begin{abstract}
Objective: Nonsteroidal anti-inflammatory drugs are considered the mainstay in the treatment of renal colic. Nonsteroidal anti-inflammatory drugs are contraindicated in patients with renal failure and are not recommended in patients with certain diseases. In these cases other analgesics should be used. The aim of our study was to compare the analgesic efficacy of intramuscular Diclofenac and Tramadol in the treatment of renal colic in our emergency department.

Methods: A prospective, randomized trial was conducted in patients with a clinical picture of renal colic. Diagnosis was confirmed by non contrast abdominal computed tomography. Subjects were randomized to receive a single intramuscular injection of either $75 \mathrm{mg}$ Diclofenac or $100 \mathrm{mg}$ Tramadol. Ninety seven patients were included, of these 48 received Diclofenac and 49 received Tramadol.

Results: Patients' characteristics including average stone size and degree of hydronephrosis were similar at enrollment. Diclofenac was significantly more effective than Tramadol in reducing the severity of pain at 30 minutes as measured on a $10-\mathrm{cm}$ visual analogue scale. Reduction of more than $50 \%$ in pain severity was observed in $64 \%$ of patients treated with Diclofenac and in $49 \%$ of patients treated with Tramadol $(P<0.05)$. More patients in the Tramadol group needed rescue analgesia: $51 \%$ vs $21(\mathrm{p}<0.05)$. For all the study variables, Diclofenac was better than Tramadol

Conclusions: Intramuscular Diclofenac as a single agent for the treatment of renal colic is more effective than intramuscular Tramadol in our patients. Intramuscular Tramadol may be an alternative when contraindications preclude the use of Diclofenac.
\end{abstract}

Keywords: Renal colic, analgesia, emergency department.

\section{INTRODUCTION}

Renal colic is a common presenting clinical problem in the emergency departments (ED). Usually it refers to symptomatic nephrolithiasis. The prevalence of renal colic in the general population is $10 \%$ [1]. The classic clinical picture is characterized by acute flank pain which sometimes fluctuates and radiates to the groin and scrotum in males [2]. The main issue in the management of these patients in the ED is sufficient pain control. Pain medications given for symptomatic relief is the only treatment needed in about $90 \%$ of patients $[3,4]$.

The failure of conservative treatment depends mainly on two parameters: stone size and stone location in the urinary tract [5]. There is a negative correlation between stone size and its spontaneous passage. Stones $>4 \mathrm{~mm}$ rarely pass alone [6]. This is also true for stones in the proximal ureter that tend to impact in the ureter and less frequently pass spontaneously as compared to stones located in the distal ureter [7].

*Address correspondence to this author at the Emergency Department Hadassah Mount Scopus, Jerusalem, Israel; Tel: 97225844111; Fax: 97225844176; E-mail: Shaden@hadassah.org.il
Pain is usually measured by the "visual analog score" (VAS) from 1 , no pain to 10 . VAS $<4$ usually refers to mild pain [8].

The pharmacological treatment for pain in patients with renal colic depends on its intensity: simple analgesia like paracetamol and dypirone helps in very mild cases. In moderate to severe pain, stronger analgesia is needed. Nonsteroid anti-inflammatory drugs (NSAIDs) and opiates are good options [9].

NSAIDs have the possible advantage of decreasing ureteral smooth muscle tone, thereby directly treating the mechanism by which pain is thought to occur [10]. NSAIDs are contraindicated in patients with renal failure and are not recommended in patients with certain diseases which could involve the kidney [11]. In these cases other analgesics should be used. Diclofenac is a common NSAID used in patients with renal colic.

Opiates as Morphine and Pethidine (meperidine, Demerol) are effective in pain management but could lead to respiratory depression or even respiratory arrest in about $10 \%$ and $1 \%$ of cases respectively. These statistics in general are not necessarily relevant to the population in our clinical trial 
[12]. Thus, patients given these drugs need close monitoring which could be a problem in a crowded ED.

Tramadol is a semi-synthetic opiate which has the advantage of less respiratory depression $(<1 \%)$ besides its good analgesic effect $[13,14]$.

Administration of analgesics by the oral route in patients with renal colic is not the most recommended route, because these patients might have nausea and because of its slower analgesic effect. So the preferred route is parenteral, either intravenously (IV) or intramuscularly (IM). The IM route has theoretically the advantage of not requiring monitoring and thus can be administered quickly even in a crowded ED. The aim of our study was to compare the efficacy of intramuscular Diclofenac and Tramadol in the treatment of renal colic in the ED.

\section{MATERIALS AND METHODS}

The study site was a university general hospital with 60,000 admissions a year to the ED. From June 2007 until January 2009, one hundred patients with a clinical diagnosis of renal colic were assigned to the study. The study was approved by the ethical Helsinki Committee of our hospital.

Inclusion criteria were at least 18 years of age (and less than 65 years), ability to provide written informed consent and confirmed diagnosis of ureteral calculus.

Exclusion criteria were: allergy to the study drugs, peptic ulcer disease, renal failure, diabetes, hypertension, pregnant and breast feeding women. Patients, who got analgesics up to six hours before admission, were also excluded due to the potential effect of masking the pains.

Every patient completed the process of informed consent, and was randomly assigned to one of the two study groups. Randomization was accomplished by using the pair or unpair last digit of the ID number. Participants were randomized and treated based upon clinical evaluation and prior to the confirmatory CT scan.

CT scans were performed with a 16-slice CT scanner (LightSpeed, GE Healthcare, and Milwaukee, WI) using standard parameters for abdominal CT. Neither oral nor IV contrast material was administered. Radiologic evaluation included the degree of hydronephrosis, maximal stone size and stone location, as these parameters thought to have correlation with the intensity of pain.

Each patient, was asked to rate the initial intensity of pain on a Visual Analogue Score (VAS) from 1 to 10 [8]. Only patients with moderate to severe pain (VAS score $\geq 4$ ) were included.

The patients were randomized to receive equi-analgesic doses of the study drugs; either IM Diclofenac $75 \mathrm{mg}$ (group 1) or IM Tramadol $100 \mathrm{mg}$ (group 2). Pain was evaluated at two points -before administration of study medication and 30 minutes after. This evaluation mostly was done by doctors that were blinded to treatment assignment.

When pain control was not achieved (less than 50\% reduction in VAS score) rescue analgesia with intravenous morphine was administered. The dose of morphine given was $0.1 \mathrm{mg} / \mathrm{kg}$.

\section{Statistical Analysis}

The sample size was calculated according to the assumption that patients treated with Diclofenac will have at least $80 \%$ good response (more than $50 \%$ in pain reduction) and that patients treated with Tramadol will have $60 \%$ good response. Accordingly the sample size was calculated to achieve a statistical power of $80 \%$ at $5 \%$ type I error with 50 subjects being required for each group. To calculate the difference; $t$ test was used for variables. For categorical variables we used the $\chi^{2}$ test. As well we used ANCOVA and logistic regression to compare the influence of few variables on VAS.

\section{RESULTS}

\section{Patients Characteristics}

Of the 100 patients who were randomized, 97 completed the study (the other 3 patients which 2 of them received Diclofenac and the other one Tramadol -could not be included in the analysis due to lack of accurate pain estimation. Forty eight patients received Diclofenac (group 1) and forty nine subjects received Tramadol (group 2).

All outcomes were evaluated during patients' stay in the ED. No significant side effects were reported in either group.

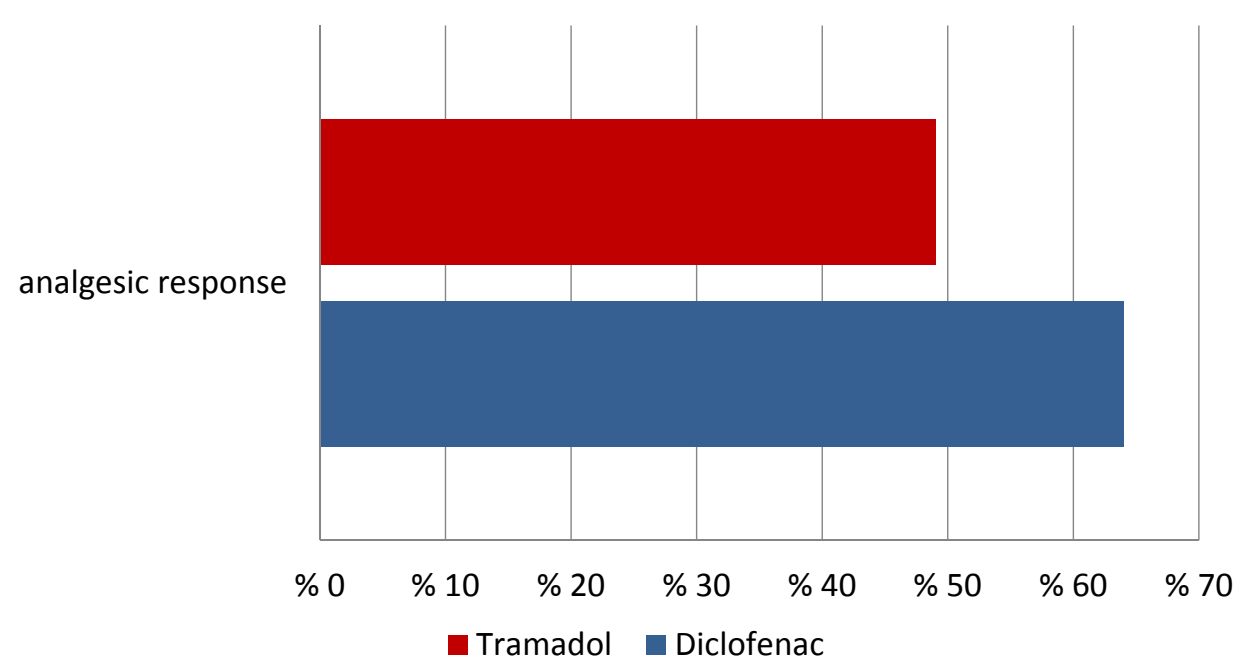

Fig. (1). The percentage of patients responded to the study drugs. 
Table 1. Averaged VAS before and after Treatment with the Study Drugs

\begin{tabular}{|c|c|c|c|}
\hline & VAS before & VAS after & The Delta Pain Reduction (\%) \\
\hline \hline Diclofenac & $8.7 \pm 1.6$ & $4.2 \pm 2.6$ & $52 \%$ \\
\hline Tramadol & $8.7 \pm 1.2$ & $5.6 \pm 2.9$ & $35 \%$ \\
\hline
\end{tabular}

Mean patient's age was $37 \pm 10$ years in group 1 and $37 \pm$ 11 years in group 2 (The total mean age: was $37 \pm 10(18-65$ years)

The Male / Female ratio was 4:1 for group 1 and 5:2 for group 2 (Total ratio 3:1).

Baseline createnine level was $92 \pm 17$ (normal range 60$110 \mathrm{mmol} / 1)$ for both groups.

The averaged VAS score at presentation was $8.7 \pm 1.6$ for group 1 and $8.7 \pm 1.2$ for group 2 (total average- $8.7 \pm 1.5$ )

\section{Pain Reduction}

Relief of pain was observed in $64 \%$ of patients treated with Diclofenac and in $49 \%$ of patients treated with Tramadol $(\mathrm{P}<0.05)$ (Fig. 1). The delta pain reduction was higher in the patients treated with Diclofenac as compared to the patients treated with Tramadol in an average of 17\% (Table 1).

Thus; more patients in the Tramadol group were needed to be treated with rescue analgesia $(\mathrm{P}<0.05)$, as shown in Fig. (2). These findings were statistically significant.

\section{Radiological Results}

The radiological analysis was done by senior radiologist according to the radiological standards. Most of the patients in both groups had mild hydronephrosis $88 \%$ and $76 \%$ in Diclofenac and Tramadol group respectively. This difference was not statistically significant (Figs. 3, 4).

Stone size was divided to two categories by a cut point of $4 \mathrm{~mm}$. a similar proportions in the two groups had stones $>$

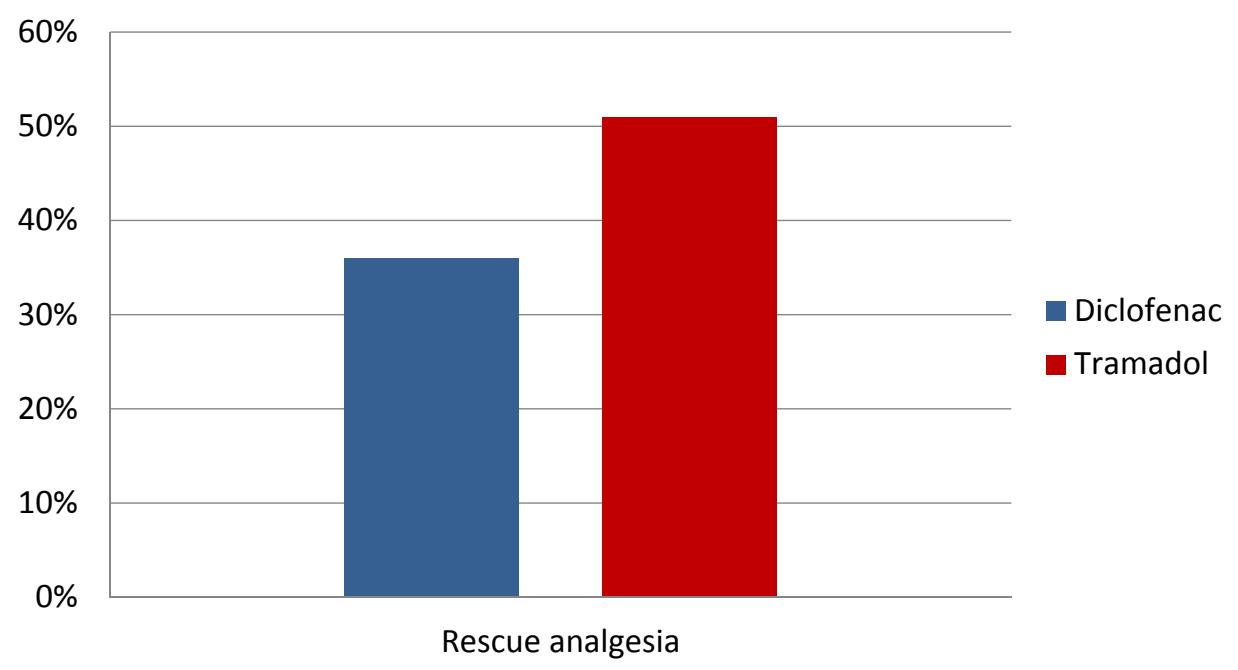

Fig. (2). The percentage of patients who needed rescue analgesia.

\section{Degree of Hydronephrosis}

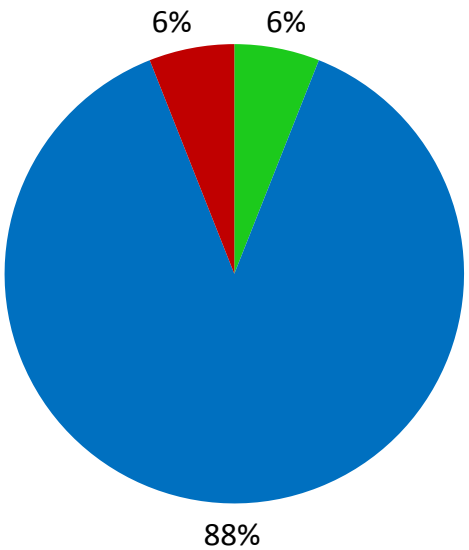

\section{Diclofenac}

No

Mild

Moderate-Severe

Fig. (3). The degree of Hydronephrosis in Diclofenac treated patients. 


\section{Degree of Hydronephrosis}

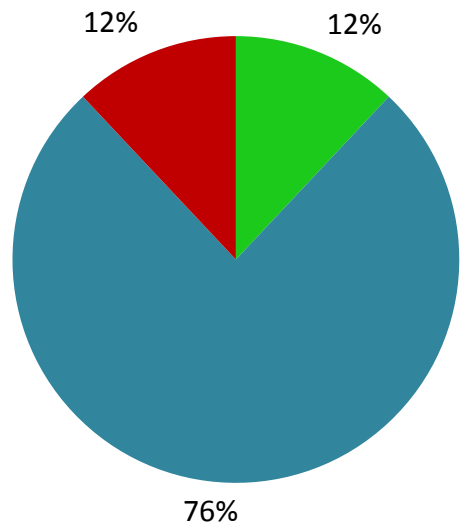

Fig. (4). The degree of Hydronephrosis in Tramadol treated patients.

$4 \mathrm{~mm}(24 \%$ vs $23 \%)$ in the Tramadol and Diclofenac group respectively.

Regarding the stone's location, the proportions of stones in the Diclofenac group were $2 \%, 54 \%, 27 \%, 17 \%$ in the uretero-pelvic junction (UPJ), ureter, uretero-vesical junction (UVJ) and bladder respectively. In the Tramadol group the proportions were: $4 \%, 47 \%, 37 \%$, and $12 \%$ in the same order. There was no statistically significant change between the two groups.

\section{DISCUSSION}

Both NSAIDs and opioids have traditionally been used for pain control in patients with acute renal colic.

Prospective randomized controlled studies suggest that NSAIDs are at least as effective as opiates $[15,16]$.

Tramadol was found effective, well tolerated agent to reduce pain resulting from renal colic. Tramadol appears to produce less constipation and respiratory depression than equi-analgesic doses of strong opioids [3].

One study evaluated the analgesic effect of Ketorolac versus Tramadol [17]. Both Ketorolac IM and Tramadol SC were found to be effective in the initial treatment of renal colic. Both drugs had an efficacy greater than $80 \%$. The analgesic effect of Ketorolac is observed earlier than that of Tramadol. This last result could be secondary to the different route of administration.

To our knowledge there were no prior studies comparing NSAIDs drug and Tramadol given in the same route in the setting of analgetic treatment in renal colic.

\section{Tramadol}

\author{
No \\ Mild \\ - Moderate-Severe
}

According to our results intramuscular Diclofenac as a single agent for the treatment of renal colic appears to be more effective than intramuscular Tramadol. The beneficial effect of Diclofenac was not influenced by the presence of hydronephrosis or its degree of severity, neither by the location or size of the calculus. All these variables were similar between both groups of treatment.

Another difference between our study and others is the way we assessed pain relief. In our study we determined response as pain reduction of at least $50 \%$ from baseline before drug administration. Other studies used the quantitative VAS score reduction [18]. More than 2 VAS score reduction from baseline was used to identify good response. We retrospectively analyzed our results according to this method. The revised results showed: response rate of $87.5 \%$ in the Diclofenac group and only $61.2 \%$ response rate in the Tramadol group. These results strengthen our original results (Table 2).

The study carries several limitations:

1) The relatively small number of research cases limits the statistical validity of some findings. A larger study would be required to examine important subgroups and complications For example. Subgroup analysis by stone size. This study was not powered enough to look at the subgroups by stone size. There would be an added benefit to repeating the study on a larger scale.

2) Time window - though the peak effect of the two drugs when given intramuscularly is similar theoretically variabilities in individual pharmacodynamics could affect the results [19].

Table 2. Quantitative VAS Score Reduction

\begin{tabular}{|c|c|c|c|}
\hline & $\begin{array}{c}\text { VAS } \\
\text { reduction>2) } \\
\mathbf{N}^{\mathbf{0}(\%)}\end{array}$ & $\begin{array}{c}\text { VAS } \\
\text { reduction <2) } \\
\mathbf{N}^{\mathbf{0}(\%)}\end{array}$ & $6(12.5 \%)$ \\
\hline \hline Diclofenac & $42(87.5 \%)$ & $19(38.8 \%)$ & $48(100 \%)$ \\
\hline Tramadol & $30(61.2 \%)$ & $25(25.8 \%)$ & $49(100 \%)$ \\
\hline Total & $72(74.2 \%)$ & $97(100 \%)$ \\
\hline
\end{tabular}


3) The study population seems to be more resistant to pain treatment - the effect of Diclofenac on pain control has been reported to be about $80 \%$ [17]. In our population the effect was only $64 \%$. So the results with Tramadol $(49 \%$-good clinical pain response) in our population may be actually better than it seems.

\section{CONCLUSIONS}

In our study, IM Diclofenac proved to be superior to Tramadol for pain relief in patients with renal colic. Tramadol could be used as an alternative when contraindications preclude the use of Diclofenac, although in our study, less than half of the subjects treated with Tramadol achieved good pain control as defined in this study.

\section{REFERENCES}

[1] Ahmed A, Abdulmaaboud M, Abdulmaaboud SH. Prospective comparison of nonenhanced helical computerized tompography and Doppler ultrasonography for the diagnosis of renal colic. J Urol 2001; 165: 1082-4.

[2] Seifter JL, Brenner BM. Urinary Tract Obstruction. Harrison principles of internal medicine; $17^{\text {th }}$ ed. Braunwald: McGraw Hill, 2008; 1827-30.

[3] Grond S, Sablotzki A. Clinical Pharmacology of Tramadol. Clin Pharmacokinet 2004; 43(13): 879-923.

[4] Eray O, Cete Y, Oktay C, et al. Intavenous single-dose tramadol versus meperidine for pain relief in renal colic. Eur J Anaesthesiol 2002; 19: 368-70.

[5] Miller OF, Kane CJ. Time to stone passage for observed ureteral calculi: a guide for patient education. J Urol 1999; 162(3): 688-90.

[6] Coll DM,Varanelli MJ, Smith RC. Relationship of spontaneous passage of ureteral calculi to stone size and location as revealed by unenhanced helical CT. Am J Roentgenol 2002; 178(1): 101-3.
[7] Parekattil SJ, Kumar U, Hegarty NJ, et al. External validation of outcome prediction model for ureteral/renal calculi. J Urol 2006; 175(2): 575-9.

[8] Gould D. Information point: Visual Analogue Scale (VAS). J Clin Nurs 2001; 10: 697-706.

[9] Holdgate A, Pollock T. Systematic review of the relative efficacy of non-steroidal anti-inflammatory drugs and opioids in the treatment of acute renal colic. BMJ 2004; 328(7453): 1401.

[10] Cole RS, Fry CH, Shuttleworth KE. The action of the prostaglandins on isolated human ureteric smooth muscle. Br J Urol 1988; 61(1): 19-26.

[11] Dieppe P, Bartlett C, Davey P, et al. Balancing benefits and harms: the example of non-steroidal anti-inflammatory drugs. BMJ 2004; 329(3): 31-4.

[12] Dahan A. Respiratory depression with opioids. J Pain Palliat Care Pharmacother 2007; 21(1): 63-6.

[13] Grond S, Sablotzki A. Clinical Pharmacology of Tramadol. Clin Pharmacokinet 2004; 43(13): 879-923.

[14] Mortelmans LJM, Desruelles D, Baert JA, et al. Use of tramadol drip in controlling renal colic pain. J Endourol 2006; 20(12): 10105.

[15] Cordell WH, Wright SW, Wolfson AB, et al. Comparison of intravenous ketorolac, meperidine, and both (balanced analgesia) for renal colic. Ann Emerg Med 1996; 28(2): 151-8.

[16] Cordell WH, Larson TA, Lingeman JE, et al. Indomethacin suppositories versus intravenously titrated morphine for the treatment of ureteral colic. Ann Emerg Med 1994; 23(2): 262-9.

[17] Nicolás TJA, Rigabert MM, Bañón PV, et al. Intramuscular ketorolac compared to subcutaneous tramadol in the initial emergency treatment of renal colic. Arch Esp Urol 1999; 52(5): 435-7.

[18] Olsen JC, McGrath NA, Schwarz DG, et al. A double-blind randomized clinical trial evaluating the analgesic efficacy of ketorolac versus butorphanol for patients with suspected biliary colic in the emergency department. Acad Emerg Med 2008; 15(8): 718-22.

[19] Charles F. Lacy, Lora L. Armstrong, Morton P. Goldman, et al. "Drug Information Handbook" A comprehensive Resource for all clinicians and Healthcare Professionals ;17th ed. :Hudson, Ohio, Lexi-Comp, 2008. 